\title{
Vestibuloplasty covering titanium mesh with grafted free gingiva on anterior mandible: technical report and rationale
}

\author{
Jeong-Kui Ku*, Dae Ho Leem \\ Department of Oral and Maxillofacial Surgery, School of Dentistry and Institute of Oral Bioscience, \\ Research Institute of Clinical Medicine of Chonbuk National University-Biomedical Research Institute of Chonbuk National University Hospital, \\ Chonbuk National University, Jeonju, Korea
}

\begin{abstract}
J Korean Assoc Oral Maxillofac Surg 2019;45:369-373)
This paper describes a patient with an insufficient vestibular depth for a removable partial denture who underwent vestibuloplasty with a free gingival graft using a titanium mesh in the anterior mandible. Free gingiva was harvested from the palatal mucosa, and a partial thickness flap was elevated at the recipient site. After minimal suturing for the graft, a titanium mesh was fixed over the graft. The mesh was removed four weeks after surgery. The patient obtained an adequate vestibular depth and keratinized gingiva eight weeks after surgery without any complications. In this case, an appropriate vestibular depth and keratinized gingiva were easily obtained by vestibuloplasty using a titanium mesh.
\end{abstract}

Key words: Gingiva, Removable partial denture, Titanium, Vestibuloplasty

[paper submitted 2019. 4. 6 / revised 2019. 5. 17 / accepted 2019. 5. 18]

\section{Introduction}

An inadequate vestibular depth (VD) in the flange region of a removable partial denture (RPD) can impede retention of the denture ${ }^{1}$. If the VD is insufficient, retention can be achieved using an implant-supported denture, but an RPD without implant support may be required for financial reasons. Vestibuloplasty is a procedure that mainly improves retention of prostheses and produces an environment where oral hygiene can be properly managed. The currently known

\footnotetext{
Dae Ho Leem

Department of Oral and Maxillofacial Surgery, School of Dentistry and Institute of Oral Bioscience, Research Institute of Clinical Medicine of Chonbuk National University-Biomedical Research Institute of Chonbuk National University Hospital, Chonbuk National University, 567 Baekjedaero, Deokjin-gu, Jeonju 54896, Korea

TEL: +82-63-250-2113 FAX: +82-63-250-2089

E-mail:idisho@jbnu.ac.kr

ORCID: https://orcid.org/0000-0001-6735-8275

*Current affiliation: Department of Oral and Maxillofacial Surgery, Section of Dentistry, Armed Forces Capital Hospital, Seongnam, Korea
}

(c) This is an open-access article distributed under the terms of the Creative Commons Attribution Non-Commercial License (http://creativecommons.org/ licenses/by-nc/4.0/), which permits unrestricted non-commercial use, distribution, and reproduction in any medium, provided the original work is properly cited.

Copyright (C) 2019 The Korean Association of Oral and Maxillofacial Surgeons. All rights reserved. vestibuloplasty techniques for obtaining VD suffer loss of obtained depth due to scar formation or continuous stimulation of the vestibule over time ${ }^{2}$.

Many studies on development of techniques to prevent relapse after vestibuloplasty have been carried out. Many modifications, such as Clark's technique, the Kazanjian technique, autogenous grafts (skin, palatal, or cheek), and allodermal and xenodermal grafts combined with autogenous grafts, have been introduced ${ }^{3}$. Skin grafts or mucosa grafts can reduce the relapse rate by $20 \%$ to $30 \%$, but a free gingival graft (FGG) is technique sensitive, so careful attention is needed to achieve successful results ${ }^{4}$. Therefore, it is essential to develop less technique-sensitive methods to effectively perform vestibuloplasty with FGG.

Titanium is used in a variety of medical fields because of its high corrosion resistance, low toxicity, very low allergenic potency, and good biocompatibility ${ }^{5}$. In general, titanium mesh is used in the submucosal region, mainly for guided bone regeneration (GBR), because of its forming force, strength, osteocyte activity of the titanium oxide layer, and biocompatibility ${ }^{6}$. In addition, titanium is used widely as a material for dental prostheses because of its ability to adapt well to the oral environment invaded by bacteria and foods with multiple acidities ${ }^{7}$. Therefore, titanium mesh can also be used in situations where it is exposed to the oral environment. 
This paper introduces a case of vestibuloplasty with a FGG performed easily and effectively in the anterior mandible titanium mesh to obtain the appropriate VD and keratinized gingiva (KG).

\section{Technical Note}

A female patient was admitted to the Emergency Department of Chonbuk National University Hospital for a \#34 to \#44 avulsion and lip penetration wound caused by a bicycle accident. A suture repair was performed. At 10 months after trauma, the patient visited the clinic for RPD fabrication. Vestibuloplasty was planned before fabrication of the RPD because of insufficient VD for retention of the RPD due to scar tissue constriction of the injured area.(Fig. 1)

Local anesthesia (Lidocaine $\mathrm{HCl}$; Huons, Seongnam, Korea) was administered at the surgical site. An approximately $3 \mathrm{~cm}$ horizontal incision was created along the margin of the remaining KG with a No. 15 blade (Braun Manufacturing, Frankfurt, Germany), and a vertical incision was made from the distal aspect of the horizontal incision toward the vestibule. A curette was used to dissect and separate the mucous membrane and the mentalis muscle from the periosteum to create a recipient site.(Fig. 2. A)

Two rectangular FGGs ( $2 \mathrm{~cm}$ long and $1 \mathrm{~cm}$ wide) were harvested from the left and right palatal mucosa using a conventional technique. The incision depth was approximately $1.5 \mathrm{~mm}$, and the graft and adipose tissues were well-separat-
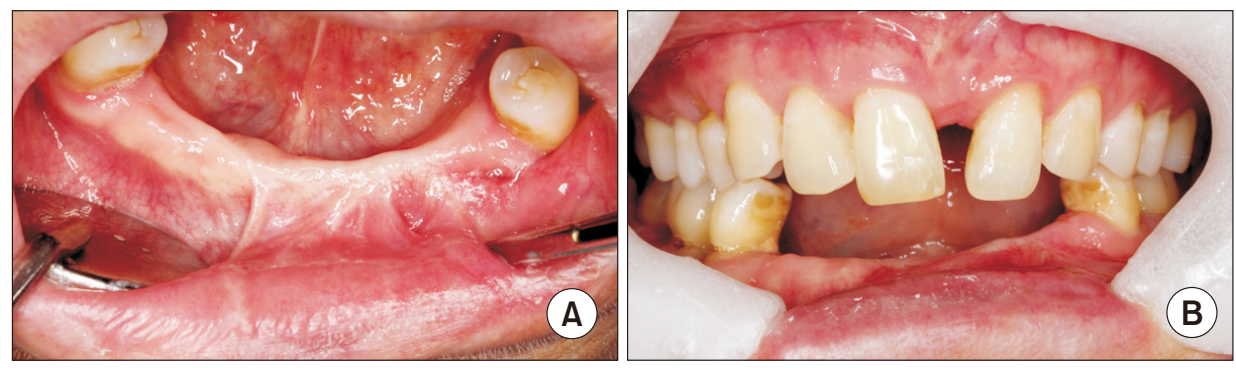

Fig. 1. Lack of vestibular depth due to scar constriction. A, B. The vestibular depth was insufficient for a removable partial denture.

Jeong-Kui Ku et al: Vestibuloplasty covering titanium mesh with grafted free gingiva on anterior mandible: technical report and rationale. J Korean Assoc Oral Maxillofac Surg 2019
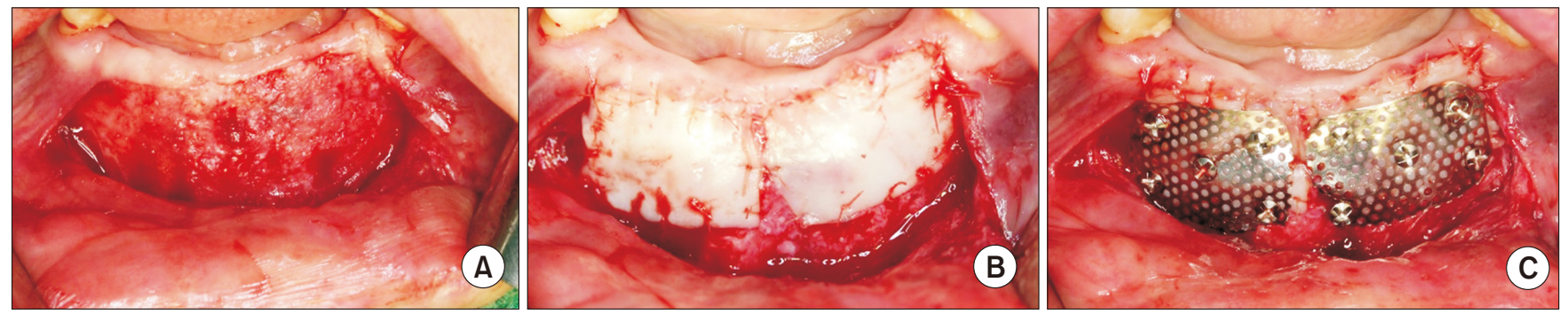

Fig. 2. Surgical procedure. A. Recipient site preparation with partial thickness flap elevation. $B$. Suturing of the free gingival grafts $(1 \times 2 \mathrm{~cm}$, from the palatal gingiva) onto the recipient periosteum. C. Adaptation of the titanium meshes with miniscrews.

Jeong-Kui Ku et al: Vestibuloplasty covering titanium mesh with grafted free gingiva on anterior mandible: technical report and rationale. J Korean Assoc Oral Maxillofac Surg 2019
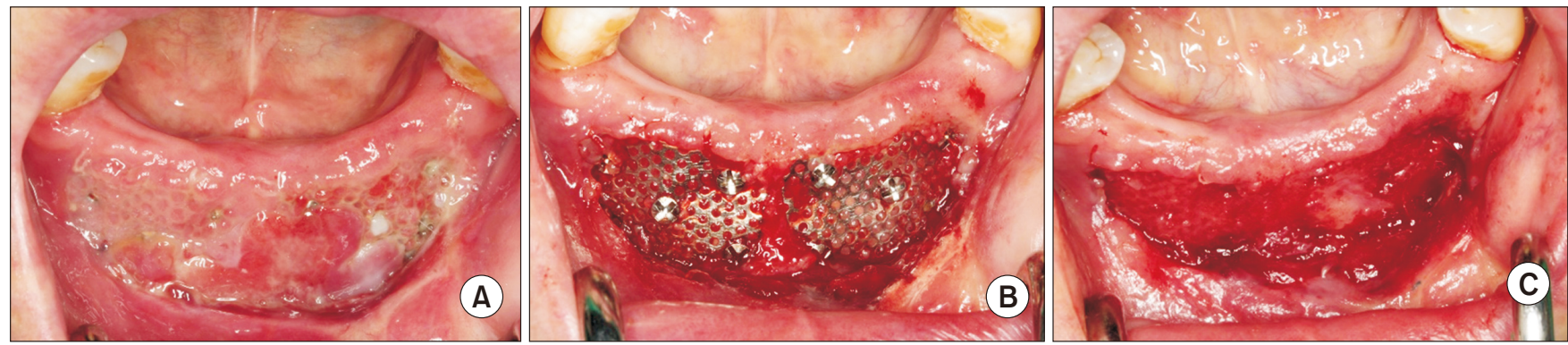

Fig. 3. Titanium mesh removal at four weeks after surgery. A. Excessive soft tissue growth covering the mesh. B. Curettage for removal of the soft tissue. C. Removal of the mesh and miniscrews.

Jeong-Kui Ku et al: Vestibuloplasty covering titanium mesh with grafted free gingiva on anterior mandible: technical report and rationale. J Korean Assoc Oral Maxillofac Surg 2019 
ed during harvest to obtain an adequate amount of mucosal tissue. The interrupted suture for the harvested graft was performed using 5-0 Vicryl (Ethicon, Somerville, NJ, USA).(Fig. 2. B) The size of the Neo Titanium mesh (depth $0.085 \mathrm{~mm}$, hole size diameter $0.4 \mathrm{~mm}$; Neo Biotech, Seoul, Korea) was adjusted to that of the graft, and the mesh was fixed above the graft using mini-screws (diameter $1.5 / 2 \mathrm{~mm}$, length $4 \mathrm{~mm}$, bone screw system; Osung MND, Gimpo, Korea).(Fig. 2. C)

Surgicel (Johnson \& Johnson, New Brunswick, NJ, USA) was applied to the palatal donor site to protect the wound, and a $0.5 \mathrm{~mm}$ Omnivec (3A MEDES, Goyang, Korea) obturator that was previously fabricated was placed on the site ${ }^{1}$.

Cephalosporin antibiotics and ibuprofen were prescribed for one week, and the patient was instructed to apply $0.1 \%$ chlorhexidine gluconate (Alpha-hexidine; Firson, Cheonan, Korea) twice a day for two weeks. The obturator was removed after five days, and the patient was provided instructions regarding a soft diet and conventional oral hygiene care. In this study, to prevent a relapse four weeks after surgery ${ }^{8}$, all the soft tissue that grew over the mesh was trimmed before removing the titanium mesh.(Fig. 3)

Four weeks after removing the mesh, normal recovery was observed, and sufficient KG and VD were obtained.(Fig. 4) At 10.3 months after surgery, the patient visited the hospital for fabrication of the RPD in the edentulous state without prosthetic treatment, and the VD was properly maintained. The average relapse rate was calculated by measuring the vertical depth from the scar margin of the gingival crest to the movable alveolar mucosa at the mesial, distal, and center of both operation sites. The relapse rate of VD was $27.38 \%$ and $24.57 \%$ on the right and left sides, respectively.

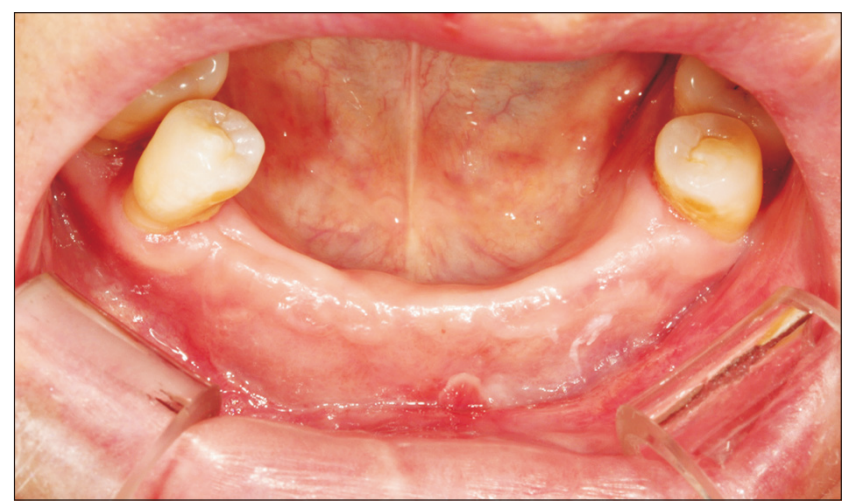

Fig. 4. Eight weeks after the operation (four weeks after mesh removal). Sufficient amounts of keratinized gingiva and vestibular depth were obtained.

Jeong-Kui Ku et al: Vestibuloplasty covering titanium mesh with grafted free gingiva on anterior mandible: technical report and rationale. J Korean Assoc Oral Maxillofac Surg 2019

\section{Discussion}

An adequate VD is an important factor for proper oral hygiene care 9 . The sequelae of various intraoral surgical approaches, such as bone grafting, implant placement, and fracture surgery, may result in loss of KG and reduced VD. If the oral vestibule is shallow or KG is insufficient, it is necessary to produce an environment suitable for retention, stability, and function of the prostheses ${ }^{10}$. Vestibuloplasty is performed when the amount of alveolar bone remaining is appropriate, and there is a lack of covering mucosal tissue, or it is unsuitable for denture support because of pathology, such as scar formation. This is a procedure to secure the prosthesis support area by preventing muscle pull and producing a deep vestibule. Unfortunately, vestibuloplasty is widely known to have a tendency for shrinkage because of the action of the adjacent muscles or scar tissue formation ${ }^{2}$. An attempt was made to reduce the relapse rate using a range of surgical techniques, including the Clark method, the Kazanjian method, the Edlan-mejchar method, and a graft with autologous, xenoderm, and alloderm materials ${ }^{11}$. In 2016, Kumar et al. ${ }^{2}$ reported that an appropriate VD was obtained using cotton rolls and a lip-periosteum mattress suture using the modified Kazanjian vestibuloplasty technique in the area of the anterior region. On the other hand, complications, such as scar formation on the lip, occurred in some patients. Therefore, technological developments are still necessary ${ }^{2}$. On the other hand, vestibuloplasty with a graft is advantageous because it reduces the incidence of relapse when there is insufficient alveolar bone or a bone defect. The level of postoperative satisfaction of patients who have undergone vestibuloplasty with a graft has been reported to be high with reduced healing period and denture placement time after surgery ${ }^{12}$. Nevertheless, many patients refuse this treatment because of the pain and discomfort at the grafting site and the need for an additional surgical site. Although many alternatives for soft tissue grafting such as living cellular construct (LCC), extracellular matrix membrane (ECM), acellular dermal matrix (ADM), bilayer collagen membrane (BCM), and LCC have been introduced, a recent systematic review has shown that they do not result in the same amount of keratinized tissue obtained through $\mathrm{FGG}^{13}$. Therefore, in this study, vestibuloplasty combined with FGG was performed to secure the VD despite the patient's discomfort. Even if vestibuloplasty using grafting is performed, relapse can occur due to graft shrinkage. In general, shrinkage of the graft occurs most frequently after one to four weeks in cases of FGG to increase the attached gingiva ${ }^{8}$. 
Therefore, in this study, an attempt was made to minimize the effects of a reduction in VD due to graft shrinkage by maintaining the titanium mesh for four weeks, when the most shrinkage occurs. The obtained VD and KG were maintained at eight weeks after vestibuloplasty, and the tissue remaining after removing the titanium mesh appeared to have undergone remodeling with the recipient site in a desirable way.

To obtain successful outcomes of FGG in general, close attention is needed to prevent dead space, hematoma formation, and instability or movement of the graft that can cause tissue necrosis through pre-operative preparation of the recipient site, suitability of the graft, and rigid fixation of the graft $^{4}$. Studies on reduction of scar tissue after grafting using the pressure of the temporary prosthesis have shown that application of uniform pressure contributes to rapid revascularization, which leads to successful grafting and minimal graft shrinkage ${ }^{14}$. On the other hand, issues such as necrosis of the graft due to excessive pressure have been reported ${ }^{15}$. In this study, stable fixation to minimize the sutures that could serve as a pathway of infection was obtained using a titanium mesh and miniscrews, while avoiding overpressure ${ }^{15}$. In addition, the surgery time could be drastically reduced by avoiding sutures for an apically positioned flap of a conventional vestibuloplasty ${ }^{2}$. Although additional surgery is necessary for mesh removal, it could provide an opportunity to separate and remove any undesired tissue from the graft site.

Titanium has many attractive properties such as corrosion resistance, low toxicity, very low allergenic potency, and good biocompatibility ${ }^{5}$. Therefore, it has been used frequently with a high rate of success in dentistry, in internal fixations and artificial joints in orthopedics, and in instruments such as pacemakers and stents in cardiovascular surgery ${ }^{5}$. Recently, it has been used widely in the form of dental implant fixtures, miniscrews, and mesh for bone grafts as a result of developments in implant dentistry. In general, titanium meshes with a thickness of $0.2 \mathrm{~mm}$ are used mainly in GBR because they are durable enough to retain the shape of grafted bones. The meshes are also flexible enough to minimize the risk of mucosal dehiscence ${ }^{16}$. In this study, because the aim of using a titanium mesh was to obtain close contact of the graft and rigid fixation, a thin mesh with a thickness of $0.085 \mathrm{~mm}$ was used to minimize the patient's discomfort. The titanium mesh located at the subgingiva for GBR does not block blood flow from the bone and mucosa because of its pores ${ }^{16}$. On the other hand, remodeling of the implanted bone is not achieved easily if the mesh is exposed to the gingiva, even if there is no inflammation in the exposed area due to titanium biocom- patibility ${ }^{17}$. This results from the pores providing pathways for external saliva and bacteria ${ }^{18}$. Few studies using titanium meshes in the oral environment have been reported because oral exposure of the titanium mesh itself is considered a complication $^{18}$. In this study, no complications were detected because a relatively thick FGG $(1.2-1.3 \mathrm{~mm})$ that is resistant to infections was applied with the titanium mesh. In addition, although patients who underwent vestibuloplasty using FGG generally suffer from pain due to exposure of the surgical site, difficulty in oral hygiene care, and difficulty in postoperative management due to delayed recovery ${ }^{19}$, the patient with mesh covering the surgical site experienced almost no discomfort or pain. Titanium can be dissolved in fluoride-containing solutions. Nevertheless, at the concentration of fluoride in commercially available toothpaste (at a fluoride concentration of approximately $1,000 \mathrm{ppm}$ ), titanium alloys do not corrode ${ }^{20}$; therefore, conventional oral self-care can be performed easily. These results suggest that the myth of exposure to the oral environment as a complication can be overcome by applying titanium mesh only to the submucosal area in GBR and applying a titanium mesh with effective biomechanical properties under various oral conditions.

In 1976, Ward ${ }^{9}$ recommended vestibuloplasty when the vestibule depth was $4 \mathrm{~mm}$ or less. There have been few studies on the criteria for VD suitable for retention of prostheses since. Recently, it was reported that a reduced VD is a risk factor for marginal bone loss around implants, loss of KG, and gingival recession ${ }^{10}$. Therefore, further studies on vestibuloplasty using a titanium mesh need to be conducted in implant patients with insufficient KG and VD.

In conclusion, this case demonstrates a successful outcome for vestibuloplasty on the anterior mandible. No complications following the vestibuloplasty using a titanium mesh were observed, and the patient did not complain of pain or discomfort. The VD produced by vestibuloplasty using titanium mesh was maintained well for eight weeks after surgery, even though no additional pressure had been applied using the prosthesis. These results suggest that a vestibuloplasty using a titanium mesh can be used effectively in clinical practice.

\section{ORCID}

Jeong-Kui Ku, https://orcid.org/0000-0003-1192-7066

Dae Ho Leem, https://orcid.org/0000-0001-6735-8275 


\section{Authors' Contributions}

J.K.K. and D.H.L. were responsible for the conception and design of the study/review/case series, and drafting and critical revision of the article. D.H.L. was responsible for data acquisition, laboratory or clinical/literature searches, and analysis and interpretation of the data collected. All authors read and approved the final manuscript.

\section{Ethics Approval and Consent to Participate}

This study was reviewed by the Chonbuk National University Hospital Institutional Review Board and received IRB review exemption (IRB No. CUH 2019-02-040).

\section{Conflict of Interest}

No potential conflict of interest relevant to this article was reported.

\section{References}

1. Deeb GR, Deeb JG. Soft tissue grafting around teeth and implants. Oral Maxillofac Surg Clin North Am 2015;27:425-48.

2. Kumar JV, Chakravarthi PS, Sridhar M, Devi KN, Kattimani VS, Lingamaneni KP. Anterior ridge extension using modified Kazanjian technique in mandible- a clinical study. J Clin Diagn Res 2016;10:ZC21-4.

3. Bhola M, Newell DH, Hancock EB. Acellular dermal allograft for vestibuloplasty--an alternative to autogenous soft tissue grafts in preprosthetic surgical procedures: a clinical report. J Prosthodont 2003;12:133-7.

4. Hatipoğlu H, Keçeli HG, Güncü GN, Sengün D, Tözüm TF. Vertical and horizontal dimensional evaluation of free gingival grafts in the anterior mandible: a case report series. Clin Oral Investig 2007;11:107-13.

5. Jorge JR, Barão VA, Delben JA, Faverani LP, Queiroz TP, Assunção WG. Titanium in dentistry: historical development, state of the art and future perspectives. J Indian Prosthodont Soc 2013;13:71-7.

6. Dorosz N, Dominiak M. Mandibular ridge reconstruction: a review of contemporary methods. Adv Clin Exp Med 2018;27:1159-68.

7. Ohkubo C, Hanatani S, Hosoi T. Present status of titanium removable dentures--a review of the literature. J Oral Rehabil 2008;35:706-14.
8. Egli U, Vollmer WH, Rateitschak KH. Follow-up studies of free gingival grafts. J Clin Periodontol 1975;2:98-104.

9. Ward VJ. A technique of measurement of the depth of the vestibular fornix in the mandibular anterior region. J Periodontol 1976;47:525-30.

10. Halperin-Sternfeld M, Zigdon-Giladi H, Machtei EE. The association between shallow vestibular depth and peri-implant parameters: a retrospective 6 years longitudinal study. J Clin Periodontol 2016;43:305-10.

11. Ephros H, Klein R, Sallustio A. Preprosthetic surgery. Oral Maxillofac Surg Clin North Am 2015;27:459-72.

12. Lim HC, An SC, Lee DW. A retrospective comparison of three modalities for vestibuloplasty in the posterior mandible: apically positioned flap only vs. free gingival graft vs. collagen matrix. Clin Oral Investig 2018;22:2121-8.

13. Dragan IF, Hotlzman LP, Karimbux NY, Morin RA, Bassir SH. Clinical outcomes of comparing soft tissue alternatives to free gingival graft: a systematic review and meta-analysis. J Evid Based Dent Pract 2017;17:370-80.e3.

14. Harrison CA, MacNeil S. The mechanism of skin graft contraction: an update on current research and potential future therapies. Burns 2008;34:153-63.

15. Fröschl T, Kerscher A. The optimal vestibuloplasty in preprosthetic surgery of the mandible. J Craniomaxillofac Surg 1997;25:85-90.

16. Yamada H, Nakaoka K, Horiuchi T, Kumagai K, Ikawa T, Shigeta Y, et al. Mandibular reconstruction using custom-made titanium mesh tray and particulate cancellous bone and marrow harvested from bilateral posterior ilia. J Plast Surg Hand Surg 2014;48:183-90.

17. Roccuzzo M, Ramieri G, Bunino M, Berrone S. Autogenous bone graft alone or associated with titanium mesh for vertical alveolar ridge augmentation: a controlled clinical trial. Clin Oral Implants Res 2007;18:286-94.

18. Rasia-dal Polo M, Poli PP, Rancitelli D, Beretta M, Maiorana C. Alveolar ridge reconstruction with titanium meshes: a systematic review of the literature. Med Oral Patol Oral Cir Bucal 2014;19:e639-46.

19. Hillerup S, Eriksen E, Solow B. Reduction of mandibular residual ridge after vestibuloplasty. A two-year follow-up study comparing the Edlan flap, mucosal and skin graft operations. Int J Oral Maxillofac Surg 1989;18:271-6.

20. Fais LM, Fernandes-Filho RB, Pereira-da-Silva MA, Vaz LG, Adabo GL. Titanium surface topography after brushing with fluoride and fluoride-free toothpaste simulating 10 years of use. J Dent 2012;40:265-75.

How to cite this article: $\mathrm{Ku} \mathrm{JK}$, Leem DH. Vestibuloplasty covering titanium mesh with grafted free gingiva on anterior mandible: technical report and rationale. J Korean Assoc Oral Maxillofac Surg 2019;45:369-373. https://doi.org/10.5125/jkaoms.2019.45.6.369 